\title{
On the existence of solutions of a nonlocal biharmonic problem
}

\section{Sur l'existence de solutions d'un problème biharmonique non local}

\author{
Khaled Kefi \\ Community College of Rafha, Northern Border University, Kingdom of Saudi Arabia \\ khaled_kefi@yahoo.fr
}

ABSTRACT. This paper is concerned with the existence of an eigenvalue for a $p(x)$-biharmonic Kirchhoff problem with Navier boundary condition. Under some suitable conditions, we establish that any $\lambda>0$ is an eigenvalue. The proofs combine variational methods with energy estimates. The main results of this paper improve and generalize the previous one introduced by Kefi and Rãdulescu (Atti Accad. Naz. Lincei Cl. Sci. Fis. Mat. Natur. 29 (2018), 439-463).

2010 Mathematics Subject Classification. 35D05, 35D30, 35J58, 35J60, 35D30, 58E05.

KEYWORDS. $p(x)$-biharmonic Kirchhoff problem, Navier boundary condition, variational principle, generalized Sobolev spaces.

\section{Introduction}

In this paper, we establish, under appropriate conditions, the existence of a weak nontrivial solution for the nonlocal $p(x)$-biharmonic problem with variable exponents

$$
\left\{\begin{array}{lll}
M(t)\left(\Delta_{p(x)}^{2} u+\beta(x)|u|^{p(x)-2} u\right) & =\lambda V(x)|u|^{q(x)-2} u, & \text { in } \Omega \\
& u=\Delta u=0, & \text { on } \partial \Omega,
\end{array}\right.
$$

where $\Omega$ is a smooth bounded domain in $\mathbb{R}^{N}(N \geq 1), M$ is a continuous function such that

$$
t:=\int_{\Omega} \frac{1}{p(x)}\left(|\Delta u|^{p(x)}+\beta(x)|u|^{p(x)}\right) d x
$$

$\lambda$ is a positive parameter while $p, q$ are continuous functions.

Note that the conditions on the function $V$ will be mentioned later.

The operator $\Delta_{p(x)}^{2} u=\Delta\left(|\Delta u|^{p(x)-2} \Delta u\right)$ is called the $p(x)$-biharmonic operator of fourth order where $p$ is a continuous non-constant function. This differential operator is a natural generalization of the $p$-biharmonic operator $\Delta_{p}^{2} u:=\Delta\left(|\Delta u|^{p-2} \Delta u\right)$, where $p>1$ is a real constant. However, the $p(x)$ biharmonic operator possesses more complicated non-linearity than the $p$-biharmonic operator, due to the fact that $\Delta_{p(x)}^{2}$ is nonhomogeneous. Note that the study of this kind of operators is very interesting in many fields like electrorheological fluids see [33], elastic mechanics see [36], stationary thermorheological viscous flows of non-Newtonian fluids, image processing see [6] and the mathematical description of the processes filtration of barotropic gas through on porous medium see [1].

Problem (11) is related to the stationary version of the Kirchhoff equation introduced by Kirchhoff in 1883 [23]. This equation is an extension of the classical d'Alembert wave equation by considering the effects of the changes in the length of the strings during the vibrations. Due to the presence of the function $M$, problem (1) is called nonlocal problem, which implies that the equations in (1) are no 
longer pointwise. This provokes some mathematical difficulties which make the study of such a problem particulary interesting.

After the work of Lions see [24], various equations of Kirchhoff type have been investigated, see [2, 8]. Moreover, Kirchhoff-type equations involving $p$-Laplacian and $p(x)$-Laplacian have been studied in many papers; see [7, 10, 13, 14, 15, 17, 27, 28, 29, 30, 34].

Finally, we mention that fourth-order elliptic equations arise in many domains like micro-electromechanical systems, surface diffusion on solids, thin film theory, flow in Hele-Shaw cells and phase field models of multiphasic systems, see [4, 12, 26]. Recent contributions concerning a fourth order elliptic problems involving the $p(x)$-biharmonic operator can be found in [16, 19, 20, 21, 22]

Note that problem (1) has been the object of study in [21]. More precisely, the authors established the existence of a continuous spectrum consisting in an interval. Inspired by the above-mentioned paper, we show, under appropriate conditions that any $\lambda>0$ is an eigenvalue for problem (1).

This paper is divided into three sections. In the second section, we recall some Mathematical Background concerning the properties of the generalized Lebesgue-Sobolev spaces and the $p(x)$-biharmonic operator which will be used to the proof of main results.

In the third section, we give some existence results of weak solutions of problem (1).

Throughout this paper, the letters $c, c_{i}, i=1,2, \ldots$ denote positive constants which may change from line to line.

\section{Mathematical Background}

To deal with the $p(x)$-biharmonic problems, we need to introduce some functional spaces $L^{p(x)}(\Omega)$, $W^{1, p(x)}(\Omega), W_{0}^{1, p(x)}(\Omega)$ and properties of the $p(x)$-biharmonic operator which we will use later. Denote by $S(\Omega)$ the set of all measurable real-valued functions defined in $\Omega$ and by

$$
C_{+}(\bar{\Omega}):=\{f: f \in C(\bar{\Omega}), f(x)>1, \text { for all } x \in \bar{\Omega}\} .
$$

Note that two measurable functions are considered as the same element of $S(\Omega)$ when they are equal almost everywhere.

For any $p \in C_{+}(\bar{\Omega})$, we denote $1<p^{-}:=\min _{x \in \bar{\Omega}} p(x) \leq p^{+}=\max _{x \in \bar{\Omega}} p(x)<\infty$ and

$$
L^{p(x)}(\Omega)=\left\{u \in S(\Omega): \int_{\Omega}|u(x)|^{p(x)} d x<\infty\right\},
$$

with the norm

$$
|u|_{p(x)}=|u|_{L^{p(x)}(\Omega)}=\inf \left\{\gamma>0: \int_{\Omega}\left|\frac{u(x)}{\gamma}\right|^{p(x)} d x \leq 1\right\} .
$$

The space $\left(L^{p(x)}(\Omega),|u|_{p(x)}\right)$ becomes a Banach space. We call it variable exponent Lebesgue space. Moreover, this space is a separable, reflexive and uniform convex Banach space; see [25, Theorems 1.6, $1.10,1.14]$. 
Clearly, when $p(x) \equiv p$, the space $L^{p(x)}(\Omega)$ is the classical Lebesgue space $L^{p}(\Omega)$ and the norm $|u|_{p(x)}$ is the standard norm $\|u\|_{L^{p}}=\left(\int_{\Omega}|u|^{p} d x\right)^{\frac{1}{p}}$ in $L^{p}(\Omega)$.

For any positive integer $k$, let

$$
W^{k, p(x)}(\Omega)=\left\{u \in L^{p(x)}(\Omega): D^{\alpha} u \in L^{p(x)}(\Omega),|\alpha| \leq k\right\},
$$

where $\alpha=\left(\alpha_{1}, \alpha_{2}, \ldots, \alpha_{N}\right)$ is a multi-index, $|\alpha|=\sum_{i=1}^{N} \alpha_{i}$ and $D^{\alpha} u=\frac{\partial^{|\alpha|} u}{\partial^{\alpha_{1}} x_{1} \ldots \partial^{\alpha} x_{N}}$. Then $W^{k, p(x)}(\Omega)$ is a separable and reflexive Banach space equipped with the norm

$$
\|u\|_{k, p(x)}=\sum_{|\alpha| \leq k}\left|D^{\alpha} u\right|_{p(x)} .
$$

The space $W_{0}^{k, p(x)}(\Omega)$ is the closure of $C_{0}^{\infty}(\Omega)$ in $W^{k, p(x)}(\Omega)$.

Let $L^{p^{\prime}(x)}(\Omega)$ be the conjugate space of $L^{p(x)}(\Omega)$ with $\frac{1}{p}+\frac{1}{p^{\prime}}=1$. Then the following Hölder-type inequality

$$
\left|\int_{\Omega} u v d x\right| \leq\left(\frac{1}{p^{-}}+\frac{1}{\left(p^{\prime}\right)^{-}}\right)|u|_{p(x)}|v|_{p^{\prime}(x)}, \text { for all } u \in L^{p(x)}(\Omega), v \in L^{p^{\prime}(x)}(\Omega)
$$

holds. Moreover, if $f_{1}, f_{2}$ and $f_{3}: \bar{\Omega} \rightarrow(1, \infty)$ are Lipschitz continuous functions such that $1 / f_{1}(x)+$ $1 / f_{2}(x)+1 / f_{3}(x)=1$, then for any $u \in L^{f_{1}(x)}(\Omega), v \in L^{f_{2}(x)}(\Omega)$ and $w \in L^{f_{3}(x)}(\Omega)$ the following inequality holds (see [11, Proposition 2.5]):

$$
\left|\int_{\Omega} u v w d x\right| \leq\left(\frac{1}{f_{1}^{-}}+\frac{1}{f_{2}^{-}}+\frac{1}{f_{3^{-}}}\right)|u|_{f_{1}(x)}|v|_{f_{2}(x)}|w|_{f_{3}(x)} .
$$

Inequality (2) and its generalized version (3) are due to Orlicz [31].

The modular on the space $L^{p(x)}(\Omega)$ is the map $\rho_{p(x)}: L^{p(x)}(\Omega) \rightarrow \mathbb{R}$ defined by

$$
\rho_{p(x)}(u):=\int_{\Omega}|u|^{p(x)} d x
$$

and satisfy the following proposition

Proposition 1. (See[25]) For all $u, v \in L^{p(x)}(\Omega)$, we have

1. $|u|_{p(x)}<1($ resp. $=1,>1) \Leftrightarrow \rho_{p(x)}(u)<1($ resp. $=1,>1)$.

2. $\min \left(|u|_{p(x)}^{p^{-}},|u|_{p(x)}^{p^{+}}\right) \leq \rho_{p(x)}(u) \leq \max \left(|u|_{p(x)}^{p^{-}},|u|_{p(x)}^{p^{+}}\right)$.

3. $\rho_{p(x)}(u-v) \rightarrow 0 \Leftrightarrow|u-v|_{p(x)} \rightarrow 0$.

Proposition 2. (See 9$]$ ) Let $p$ and $q$ be measurable functions such that $p \in L^{\infty}(\Omega)$, and $1 \leq p(x) q(x) \leq$ $\infty$, for a.e. $x \in \Omega$. Let $u \in L^{q(x)}(\Omega), u \neq 0$. Then

$$
\min \left(|u|_{p(x) q(x)}^{p^{+}},|u|_{p(x) q(x)}^{p^{-}}\right) \leq\left.\left.|| u\right|^{p(x)}\right|_{q(x)} \leq \max \left(|u|_{p(x) q(x)}^{p^{-}},|u|_{p(x) q(x)}^{p^{+}}\right) .
$$


Definition 1. Assume that spaces $E, F$ are Banach spaces, we define the norm on the space $X:=E \cap F$ as $\|u\|_{X}=\|u\|_{E}+\|u\|_{F}$.

In order to discuss problem (1), we need some properties of the space $X:=W_{0}^{1, p(x)}(\Omega) \cap W^{2, p(x)}(\Omega)$. From Definition 1, we know that for any $u \in X$ we have $\|u\|=\|u\|_{1, p(x)}+\|u\|_{2, p(x)}$, thus $\|u\|=$ $|u|_{p(x)}+|\nabla u|_{p(x)}+\sum_{|\alpha|=2}\left|D^{\alpha} u\right|_{p(x)}$. In Zang and Fu [35], the equivalence of the norms was proved, and it was even proved that the norm $|\Delta u|_{p(x)}$ is equivalent to the norm $\|u\|$ (see [35, Theorem 4.4]). Note that $(X,\|\|$.$) is a separable and reflexive Banach space.$

Let

$$
\|u\|_{\beta}:=\inf \left\{\mu>0: \int_{\Omega}\left(\left|\frac{\Delta u}{\mu}\right|^{p(x)}+\beta(x)\left|\frac{u}{\mu}\right|^{p(x)}\right) d x \leq 1\right\} \quad \text { for } u \in X .
$$

Since $\beta \in L^{\infty}(\Omega)$ and $\operatorname{essinf}_{x \in \Omega} \beta>0$, we deduce that $\|u\|_{\beta}$ is equivalent to the norms $\|u\|$ and $|\Delta u|_{p(x)}$ in $X$. In our paper, we will use the norm $\|u\|_{\beta}$ and the modular is defined as $\rho_{p(x)}: X \rightarrow \mathbb{R}$ by

$$
\rho_{p(x)}(u)=\int_{\Omega}\left(|\Delta u|^{p(x)}+\beta(x)|u|^{p(x)}\right) d x,
$$

which satisfies the same properties as Proposition 1. Accordingly, we have the following property.

Proposition 3. For all $u \in L^{p(x)}(\Omega)$, we have

1. $\|u\|_{\beta}<1($ resp. $=1,>1) \Leftrightarrow \rho_{p(x)}(u)<1($ resp. $=1,>1)$.

2. $\min \left(\|u\|_{\beta}^{p^{-}},\|u\|_{\beta}^{p^{+}}\right) \leq \rho_{p(x)}(u) \leq \max \left(\|u\|_{\beta}^{p^{-}},\|u\|_{\beta}^{p^{+}}\right)$.

3. $\left\|u_{n}\right\|_{\beta} \rightarrow 0$ (respectively, $\left.\rightarrow \infty\right) \Leftrightarrow \rho_{p(x)}\left(u_{n}\right) \rightarrow 0$ (respectively, $\rightarrow \infty$ ).

Proposition 4. Let $L(u)=\int_{\Omega} \frac{1}{p(x)}\left(|\Delta u|^{p(x)}+\beta(x)|u|^{p(x)}\right) d x$, then

1. $L: X \rightarrow \mathbb{R}$ is sequentially weakly lower semi continuous, $L \in C^{1}(X, \mathbb{R})$.

2. The mapping $L^{\prime}: X \rightarrow X^{*}$ is a strictly monotone, bounded homeomorphism and is of type $\left(S_{+}\right)$. That is, if $u_{n} \rightarrow u$ and $\lim \sup L^{\prime}\left(u_{n}\right)\left(u_{n}-u\right) \leq 0$, then $u_{n} \rightarrow u$.

Finally, we recall that the critical Sobolev exponent is defined as follows

$$
p_{2}^{*}(x)= \begin{cases}\frac{N p(x)}{N-2 p(x)}, & p(x)<\frac{N}{2}, \\ +\infty, & p(x) \geq \frac{N}{2} .\end{cases}
$$

It is known, for $q \in C^{+}(\bar{\Omega})$ with $q(x)<p_{2}^{*}(x)$ for all $x \in \bar{\Omega}$, that the injection $X \hookrightarrow L^{q(x)}(\Omega)$ is compact and continuous. 
In this work the function $M$ satisfy the following conditions:

$\left(H_{0}\right) M: \mathbb{R} \rightarrow\left[m_{0},+\infty\right)$ is a continuous function, with $m_{0}>0$

$\left(H_{1}\right)$ there exists $0<\theta<1$ such that

$$
\widehat{M}(t) \geq(1-\theta) M(t) t \text { for all } t \geq 0, \text { where } \widehat{M}(t)=\int_{0}^{t} M(s) d s
$$

\section{Auxiliary and main results for problem (1)}

Definition 2. We say that $\lambda \in \mathbb{R}$ is an eigenvalue of problem (1), if there exists $u \in X \backslash\{0\}$ such that $\Delta u=0$ on $\partial \Omega$ and

$$
\begin{array}{r}
M\left(\int_{\Omega} \frac{1}{p(x)}\left(|\Delta u|^{p(x)}+\beta(x)|u|^{p(x)}\right) d x\right) \int_{\Omega}\left(|\Delta u|^{p(x)-2} \Delta u \Delta v+\beta(x)|u|^{p(x)-2} u v\right) d x= \\
\lambda \int_{\Omega} V(x)|u|^{q(x)-2} u v d x
\end{array}
$$

for any $v \in X$. Moreover, if $\lambda$ is an eigenvalue of problem (1), then the corresponding $u \in X \backslash\{0\}$ is a weak solution of problem (1).

In the sequel and in order to prove our main results, we recall the following Theorem

Theorem 1. (see[5]) Let $g$ be a continuous function defined on all of $\mathbb{R}^{N}$. If $g$ is coercive, then $g$ has a global minimizer. Furthermore, if the first partial derivatives of $g$ exists on all $\mathbb{R}^{N}$, then any global minimizers of $g$ can be founded among the critical points of $g$.

\section{1. $V: \Omega \rightarrow \mathbb{R}$ is a sign-changing function and $V \in L^{\infty}(\Omega)$}

In this section, we consider problem (11) in the case when $V: \Omega \rightarrow \mathbb{R}$ is a sign-changing function. More precisely, we assume that the function $V \in L^{\infty}(\Omega)$ satisfies

$\left(V_{2}\right)$ there exist an $x_{0} \in \Omega$ and two positive constants $r$ and $R$ with $0<r<R$ such that $\overline{B_{R}\left(x_{0}\right)} \subset \Omega$ and $V(x)=0$ for $x \in \overline{B_{R}\left(x_{0}\right) \backslash B_{r}\left(x_{0}\right)}$ and one of the following conditions hold

$$
V(x)>0, \forall x \in B_{r}\left(x_{0}\right) \text { and } V(x)<0, \forall x \in \Omega \backslash \overline{B_{R}\left(x_{0}\right)}
$$

or

$$
V(x)<0, \forall x \in B_{r}\left(x_{0}\right) \text { and } V(x)>0, \forall x \in \Omega \backslash \overline{B_{R}\left(x_{0}\right)} .
$$

Moreover, we assume that

$\left(Q_{1}\right) 1<q(x)<p_{2}^{*}(x)=\frac{N p(x)}{N-2 p(x)}$ for all $x \in \Omega$ 
$\left(Q_{2}\right)$ Either

$$
\max _{x \in \overline{B_{r}\left(x_{0}\right)}} q(x)<p^{-} \leq p^{+}<\min _{x \in \overline{\Omega \backslash B_{R}\left(x_{0}\right)}} q(x)
$$

or

$$
\max _{x \in \overline{\Omega \backslash B_{R}\left(x_{0}\right)}} q(x)<p^{-} \leq p^{+}<\min _{x \in \overline{B_{r}\left(x_{0}\right)}} q(x) .
$$

We can see that there are many functions satisfying the conditions $\left(V_{2}\right),\left(Q_{1}\right)$ and $\left(Q_{2}\right)$, for that we refer the reader to the work of B. Ali et Al [3]. Our first result is the following

Theorem 2. Assume that the conditions $\left(H_{0}\right),\left(H_{1}\right),\left(V_{2}\right)$ and $\left(Q_{1}\right)$ are satisfied. Moreover, either the conditions $\left(V_{2}^{\prime}\right)-\left(Q_{2}^{\prime}\right)$ or the conditions $\left(V_{2}^{\prime \prime}\right)-\left(Q_{2}^{\prime \prime}\right)$ hold. Then any $\lambda>0$ is an eigenvalue of problem (1).

For any $\lambda>0$, let us define the functional $\Psi_{\lambda}(u): X \rightarrow \mathbb{R}$ by

$$
\Psi_{\lambda}(u)=\widehat{M}\left(\int_{\Omega} \frac{1}{p(x)}\left(|\Delta u|^{p(x)}+\beta(x)|u|^{p(x)}\right) d x\right)-\lambda \int_{\Omega} \frac{V(x)}{q(x)}|u|^{q(x)} d x,
$$

then by the hypothesis $\left(Q_{1}\right)$ and the continuous embedding of $X$ in $L^{q(x)}$, we can show that the functional $\Psi_{\lambda}$ is well-defined on $X$ and $\Psi_{\lambda} \in C^{1}(X, \mathbb{R})$ with the derivative given by

$$
\begin{array}{r}
\Psi_{\lambda}^{\prime}(u)(v)=M\left(\int_{\Omega} \frac{1}{p(x)}\left(|\Delta u|^{p(x)}+\beta(x)|u|^{p(x)}\right) d x\right) \int_{\Omega}\left(|\Delta u|^{p(x)-2} \Delta u \Delta v+\beta(x)|u|^{p(x)-2} u v\right) d x \\
-\lambda \int_{\Omega} V(x)|u|^{q(x)-2} u v d x
\end{array}
$$

for all $u, v \in X$. Therefore, the weak solutions of problem (1) are exactly the critical points of the functional $\Psi_{\lambda}$. For the proof of this result we refer to the work of Kefi and Rãdulescu (See Proposition 5 in[21]).

In order to prove Theorem 2, Let us define the functions $q_{1}$ and $q_{2}$ as follows $q_{1}: \overline{B_{r}}\left(x_{0}\right) \rightarrow(1,+\infty), q_{1}(x)=$ $q(x)$ for any $x \in \overline{B_{r}\left(x_{0}\right)}$ and $q_{2}: \overline{\Omega \backslash B_{R}\left(x_{0}\right)} \rightarrow(1,+\infty), q_{2}(x)=q(x)$ for any $x \in \overline{\Omega \backslash B_{R}\left(x_{0}\right)}$. We also introduce here the notations

$$
\begin{aligned}
& q_{1}^{-}=\underset{x \in \frac{\min }{B_{r}\left(x_{0}\right)}}{ } q_{1}(x), \quad q_{1}^{+}=\max _{x \in \overline{B_{r}\left(x_{0}\right)}} q_{1}(x) \\
& q_{2}^{-}=\underset{x \in \frac{\min }{\Omega \backslash B_{R}\left(x_{0}\right)}}{ } q_{2}(x), \quad q_{2}^{+}={ }_{x \in \frac{\max }{\Omega \backslash B_{R}\left(x_{0}\right)}} q_{2}(x) .
\end{aligned}
$$

By the conditions $\left(Q_{1}\right)$ and $\left(Q_{2}^{\prime}\right)$

$$
1<q_{1}^{-} \leq q_{1}^{+}<p^{-} \leq p^{+}<q_{2}^{-} \leq q_{2}^{+}<p_{2}^{*}(x) \text { for all } x \in \bar{\Omega},
$$

hence, for $i=1,2, X$ is continuously embedded in $L^{q_{i}}(\Omega)$, we deduce that there exists a positive constant $c_{i}$ such that

$$
|u|_{L^{q_{i}(\Omega)}} \leq c_{i}\|u\|_{\beta}, \quad \text { for all } u \in X \text { and } i=1,2 .
$$

The following result asserts the existence of a "valley" for $\Psi_{\lambda}$ near the origin. 
Lemma 1. There exists $\varphi \in X$, such that $\varphi \geq 0, \varphi \neq 0$, and $\Psi_{\lambda}(t \varphi)<0$ for any $t>0$ small enough.

Proof. By hypothesis $\left(H_{1}\right)$, there exists $t_{0}>0$ such that for all $t>t_{0}$, we have

$$
\widehat{M}(t) \leq \frac{\widehat{M}\left(t_{0}\right)}{t_{0}^{\frac{1}{1-\theta}}} t^{\frac{1}{1-\theta}}=c_{3} t^{\frac{1}{1-\theta}}
$$

Let $\varphi \in C_{0}^{\infty}(\Omega), \varphi \geq 0$ and there exist $x_{1} \in B_{r}\left(x_{0}\right)$ and $\epsilon>0$ such that for any $x \in B_{\epsilon}\left(x_{1}\right) \subset B_{r}\left(x_{0}\right)$ we have $\varphi(x)>0$. Letting $0<t<1$ we then obtain

$$
\begin{aligned}
\Psi_{\lambda}(t \varphi) & =\widehat{M}\left(\int_{\Omega} \frac{1}{p(x)}\left(|\Delta(t \varphi)|^{p(x)}+\beta(x)|t \varphi|^{p(x)}\right) d x\right)-\lambda \int_{\Omega} \frac{V(x)}{q(x)}|t \varphi|^{q(x)} d x \\
& \leq c_{3}\left(\int_{\Omega} \frac{t^{p(x)}}{p(x)}\left(|\Delta \varphi|^{p(x)}+\beta(x)|\varphi|^{p(x)}\right) d x\right)^{\frac{1}{1-\theta}}-\lambda \int_{B_{r}\left(x_{0}\right)} \frac{V(x)}{q_{1}(x)} t^{q_{1}(x)}|\varphi|^{q_{1}(x)} d x \\
& \leq \frac{t^{\frac{p^{-}}{1-\theta}}}{\left(p^{-}\right)^{\frac{1}{1-\theta}}}\left(\int_{\Omega}\left(|\Delta \varphi|^{p(x)}+\beta(x)|\varphi|^{p(x)}\right) d x\right)^{\frac{1}{1-\theta}}-\frac{\lambda t^{q_{1}^{+}}}{q_{1}^{+}} \int_{B_{r}\left(x_{0}\right)} V(x)|\varphi|^{q_{1}(x)} d x \\
& \leq c_{4} t^{p^{-}}\left(\int_{\Omega}\left(|\Delta \varphi|^{p(x)}+\beta(x)|\varphi|^{p(x)}\right) d x\right)^{\frac{1}{1-\theta}}-\frac{\lambda t^{q_{1}^{+}}}{q_{1}^{+}} \int_{B_{\epsilon\left(x_{1}\right)}} V(x)|\varphi|^{q_{1}(x)} d x
\end{aligned}
$$

Obviously, we have $\Psi_{\lambda}(t \varphi)<0$ for any $0<t<\delta^{\frac{1}{p^{-}-q_{1}^{+}}}$, where

$$
0<\delta<\min \left\{1, \frac{\frac{\lambda}{c_{4} q_{1}^{+}} \int_{B_{\epsilon}\left(x_{1}\right)} V(x)|\varphi|^{q_{1}(x)} d x d x}{\left(\int_{\Omega}\left(|\Delta \varphi|^{p(x)}+\beta(x)|\varphi|^{p(x)}\right) d x\right)^{\frac{1}{1-\theta}}}\right\} .
$$

Finally, we point out that

$$
\int_{\Omega}\left(|\Delta \varphi|^{p(x)}+\beta(x)|\varphi|^{p(x)}\right) d x>0 .
$$

Indeed, supposing the contrary we have $\int_{\Omega}\left(|\Delta \varphi|^{p(x)}+\beta(x)|u|^{p(x)}\right) d x=0$.

By Proposition 3, we deduce that $\|\varphi\|_{\beta}=0$ and consequently $\varphi=0$ in $\Omega$ which is a contradiction. The proof of Lemma1 is complete.

Proof of Theorem 2. We will prove Theorem 2 in details for the case when the conditions $\left(V_{2}^{\prime}\right)-\left(Q_{2}^{\prime}\right)$ hold, the remaining one can be made by similarly arguments so we omit it. Using Hölder inequality (2) for $\|u\|_{\beta}>1$ combined with relations (5), it follows for any $\lambda>0$ and all $u \in X$ with $\|u\|_{\beta}>1$,

$$
\Psi_{\lambda}(u)=\widehat{M}\left(\int_{\Omega} \frac{1}{p(x)}\left(|\Delta u|^{p(x)}+\beta(x)|u|^{p(x)}\right) d x\right)-\lambda \int_{\Omega} \frac{V(x)}{q(x)}|u|^{q(x)} d x
$$




$$
\begin{aligned}
& \geq \frac{m_{0}}{p^{+}}\|u\|_{\beta}^{p^{-}}-\frac{\lambda}{q^{-}} \int_{\Omega} V(x)|u|^{q(x)} d x \\
& \geq \frac{m_{0}}{p^{+}}\|u\|_{\beta}^{p^{-}}-\frac{\lambda}{q^{-}}|V|_{\infty} \int_{B_{r}\left(x_{0}\right)}|u|^{q_{1}(x)} d x \\
& \geq \frac{m_{0}}{p^{+}}\|u\|_{\beta}^{p^{-}}-\frac{\lambda}{q^{-}}|V|_{\infty} \max \left(c_{1}^{q_{1}^{-}}\|u\|_{\beta}^{q_{1}^{-}}, c_{1}^{q_{1}^{+}}\|u\|_{\beta}^{q_{1}^{+}}\right) .
\end{aligned}
$$

By $\left(Q_{2}^{\prime}\right)$, we have $q_{1}^{+}<p^{-}$, then $\Psi_{\lambda}(u) \rightarrow+\infty$, as $\|u\|_{\beta} \rightarrow+\infty$. This implies that $\Psi_{\lambda}$ is coercive and bounded from below on $X$. On the other hand, by $\left(Q_{1}\right)$, the embedding $X \hookrightarrow L^{q(x)}(\Omega)$ is compact, so $\Psi_{\lambda}$ is weakly lower semicontinuous then it has a global minimizer $w$. Due to Theorem 1, $w$ is weak solution of problem (1). However, we must show that $\Delta w=0$ on $\partial \Omega$. For this result, we encourage the reader to refer to the work [20, 21]. Finally, we point out that due to the Lemma 1, this minimizer is nontrivial and thus any $\lambda>0$ is an eigenvalue of problem (1). Which ends the proof.

\section{2. $V: \Omega \rightarrow \mathbb{R}$ is a sign-changing function and $V \in L^{s(x)}(\Omega)$}

In this part, we study problem (1) under the following assumptions

$\left(H_{2}\right) 1<q(x)<p(x)<\frac{N}{2}<s(x)$, for all $x \in \bar{\Omega}, V \in L^{s(x)}(\Omega)$ and $V>0$ in $\Omega_{0} \subset \subset \Omega$, with $\left|\Omega_{0}\right|>0$. We point out that Kefi in [18] was the first to introduce assumption like $\left(H_{2}\right)$ to study problems involving Lebesgue and Sobolev spaces with variable exponents.

Let $s^{\prime}(x)$ the conjugate exponent of the function $s(x)$ and set $r(x):=\frac{s(x) q(x)}{s(x)-q(x)}$.

We announce than have the following remark

Remark 1. Assume that assumption $\left(H_{2}\right)$ is fulfilled, then $\max \left(r(x), s^{\prime}(x) q(x)\right)<p_{2}^{*}(x)$, for all $x \in \bar{\Omega}$, consequently the embeddings $X \hookrightarrow L^{s^{\prime}(x) q(x)}(\Omega)$ and $X \hookrightarrow L^{r(x)}(\Omega)$ are compact and continuous.

Our main result in this part is the following

Theorem 3. Assume that hypotheses $\left(H_{0}\right),\left(H_{1}\right),\left(H_{2}\right)$ are fulfilled. Then, for any $\lambda>0$ problem (1) has a weak solution with negative energy.

In order to describe the variational framework associated to (1), we define the functionals $\Phi, J: X \rightarrow$ $\mathbb{R}$ defined as follows:

$$
\Phi(u)=\widehat{M}\left(\int_{\Omega} \frac{1}{p(x)}\left(|\Delta u|^{p(x)}+\beta(x)|u|^{p(x)}\right) d x\right) \text { and } J(u)=\int_{\Omega} \frac{V(x)}{q(x)}|u|^{q(x)} d x .
$$

By Proposition 2 and Remark $1, J$ is well defined and for all $u \in X$

$$
|J(u)| \leq\left.\left.\frac{1}{q^{-}}|V|_{s(x)}|| u\right|^{q(x)}\right|_{s^{\prime}(x)} \leq \begin{cases}\frac{1}{q^{-}}|V|_{s(x)}|u|_{s^{\prime}(x) q(x)}^{q^{-}}, & \text {if }|u|_{s^{\prime}(x) q(x)} \leq 1 \\ \frac{1}{q^{-}}|V|_{s(x)}|u|_{s^{\prime}(x) q(x)}^{q^{+}}, & \text {if }|u|_{s^{\prime}(x) q(x)}>1 .\end{cases}
$$

We recall that the Euler-Lagrange functional corresponding to problem (1) can be written as

$$
\Psi_{\lambda}(u):=\Phi(u)-\lambda J(u) .
$$




\subsection{Proof of Theorem 3}

We start with the following auxiliary property.

Proposition 5. Assume that hypotheses $\left(H_{0}\right),\left(H_{1}\right)$ and $\left(H_{2}\right)$ are fulfilled. Then $\Psi_{\lambda} \in C^{1}(X, \mathbb{R})$ is weakly lower semi-continuous and $u \in X$ is a critical point of $\Psi_{\lambda}$ if and only if $u$ is a weak solution of problem (1).

Proof. we chose to omit the proof since it is the same as that presented in the work of Kefi and Rãdulescu (See Proposition 5 in[21]).

In order to prove our result, we start by announcing the following Lemma

Lemma 2. Suppose that the hypotheses of Theorem 3 are fulfilled. Then $\Psi_{\lambda}$ is coercive in $X$

Proof. Since the embedding $X \hookrightarrow L^{s^{\prime}(x) q(x)}(\Omega)$ is continuous, we have

$$
|u|_{s^{\prime}(x) q(x)} \leq c_{2}\|u\|_{\beta}, \text { for all } u \in X
$$

where $c_{2}$ is the positive constant. Moreover, by hypothesis $\left(H_{0}\right)$, we have $\widehat{M}(t) \geq m_{0} t$. In what follows, let us assume that that $\|u\|_{\beta}>1$, by combining Hölder's inequality (2), Proposition 3 and inequality (6), we deduce that for all $u \in X$ with $\|u\|_{\beta}>1$

$$
\begin{aligned}
\Psi_{\lambda}(u) & =\widehat{M}\left(\int_{\Omega} \frac{1}{p(x)}\left(|\Delta u|^{p(x)}+\beta(x)|u|^{p(x)}\right) d x\right)-\frac{\lambda}{q^{-}} \int_{\Omega} V(x)|u|^{q(x)} d x \\
& \geq \frac{m_{0}}{p^{+}}\|u\|_{\beta}^{p^{-}}-\left.\left.\frac{\lambda}{q^{-}}|V|_{s(x)}|| u\right|^{q(x)}\right|_{s^{\prime}(x)} \\
& \geq \frac{m_{0}}{p^{+}}\|u\|_{\beta}^{p^{-}}-\frac{\lambda}{q^{-}}|V|_{s(x)} \max \left(|u|_{s^{\prime}(x) q(x)}^{q^{-}},|u|_{s^{\prime}(x) q(x)}^{q^{+}}\right) \\
& \geq \frac{m_{0}}{p^{+}}\|u\|_{\beta}^{p^{-}}-\frac{\lambda}{q^{-}}|V|_{s(x)} \max \left(c_{2}^{q^{-}}\|u\|_{\beta}^{q^{-}}, c_{2}^{q^{+}}\|u\|_{\beta}^{q^{+}}\right)
\end{aligned}
$$

Since $q^{+}<p^{-}$, then $\Psi_{\lambda, \beta}(u) \rightarrow+\infty$, as $\|u\|_{\beta} \rightarrow+\infty$, consequently $\Psi_{\lambda}$ is coercive. which ends the proof.

The following result asserts the existence of a valley for $\Psi_{\lambda}$ near the origin.

Lemma 3. There exists $\varphi \in X \backslash\{0\}$ such that $\varphi \geq 0$ and $\Psi_{\lambda}(t \varphi)<0$, for $t>0$ small enough.

Proof. By hypothesis $\left(H_{1}\right)$, there exists $t_{0}>0$ such that for all $t>t_{0}$, we have

$$
\widehat{M}(t) \leq \frac{\widehat{M}\left(t_{0}\right)}{t_{0}^{\frac{1}{1-\theta}}} t^{\frac{1}{1-\theta}}=c_{3} t^{\frac{1}{1-\theta}}
$$

Moreover, by $\left(H_{2}\right)$, we have $q(x)<p(x)$, for all $x \in \bar{\Omega}_{0}$. 
In the sequel, we denote

$$
q_{0}^{-}:=\inf _{\Omega_{0}} q(x) \quad \text { and } \quad p_{0}^{-}:=\inf _{\Omega_{0}} p(x) .
$$

Let $\epsilon_{0}$ be such that $q_{0}^{-}+\epsilon_{0}<p_{0}^{-}$. Since $q \in C\left(\bar{\Omega}_{0}\right)$, there exists an open set $\Omega_{1} \subset \Omega_{0}$ such that $\left|q(x)-q_{0}^{-}\right|<\epsilon_{0}$, for all $x \in \Omega_{1}$. It follows that $q(x) \leq q_{0}^{-}+\epsilon_{0}<p_{0}^{-}$, for all $x \in \Omega_{1}$.

Let $\varphi \in C_{0}^{\infty}(\Omega)$ be such that $\operatorname{supp}(\varphi) \subset \Omega_{1} \subset \Omega_{0}, \varphi=1$ in a subset $\Omega_{1}^{\prime} \subset \operatorname{supp}(\varphi)$, and $0 \leq \varphi \leq 1$ in $\Omega_{1}$. It follows that

$$
\begin{aligned}
\Psi_{\lambda}(t \varphi) & =\widehat{M}\left(\int_{\Omega} \frac{1}{p(x)}\left(|\Delta(t \varphi)|^{p(x)}+\beta(x)|t \varphi|^{p(x)}\right) d x\right)-\lambda \int_{\Omega} \frac{V(x)}{q(x)}|t \varphi|^{q(x)} d x \\
& \leq c_{3}\left(\int_{\Omega_{0}} \frac{t^{p(x)}}{p(x)}\left(|\Delta \varphi|^{p(x)}+\beta(x)|\varphi|^{p(x)}\right) d x\right)^{\frac{1}{1-\theta}}-\lambda \int_{\Omega_{1}} \frac{V(x)}{q(x)} t^{q(x)} V(x)|\varphi|^{q(x)} d x \\
& \leq \frac{t^{\frac{p_{0}^{-}}{1-\theta}}}{\left(p_{0}^{-}\right)^{\frac{1}{1-\theta}}}\left(\int_{\Omega_{0}}\left(|\Delta \varphi|^{p(x)}+\beta(x)|\varphi|^{p(x)}\right) d x\right)^{\frac{1}{1-\theta}}-\frac{\lambda t^{q_{0}^{-}+\epsilon_{0}}}{q_{0}^{+}} \int_{\Omega_{1}} V(x)|\varphi|^{q(x)} d x \\
& \leq c_{4} t^{p_{0}^{-}}\left(\int_{\Omega_{0}}\left(|\Delta \varphi|^{p(x)}+\beta(x)|\varphi|^{p(x)}\right) d x\right)^{\frac{1}{1-\theta}}-\frac{\lambda t^{q_{0}^{-}+\epsilon_{0}}}{q_{0}^{+}} \int_{\Omega_{1}} V(x)|\varphi|^{q(x)} d x .
\end{aligned}
$$

Therefore

$$
\Psi_{\lambda}(t \varphi)<0
$$

for $t<\delta^{1 /\left(p_{0}^{-}-q_{0}^{-}-\epsilon_{0}\right)}$ with

$$
0<\delta<\min \left\{1, \frac{\frac{\lambda}{c_{4} q_{0}^{+}} \int_{\Omega_{1}} V(x)|\varphi|^{q(x)} d x}{\left(\int_{\Omega_{0}}\left(|\Delta \varphi|^{p(x)}+\beta(x)|\varphi|^{p(x)}\right) d x\right)^{\frac{1}{1-\theta}}}\right\} .
$$

Finally, we point out that

$$
\int_{\Omega_{0}}\left(|\Delta \varphi|^{p(x)}+\beta(x)|\varphi|^{p(x)}\right) d x>0
$$

Indeed, supposing the contrary we have $\int_{\Omega}\left(|\Delta \varphi|^{p(x)}+\beta(x)|u|^{p(x)}\right) d x=0$. By Proposition $\underline{3}$, we deduce that $\|\varphi\|_{\beta}=0$ and consequently $\varphi=0$ in $\Omega$ which is not true. The proof of Lemma 3 is complete.

Proof of Theorem 3 completed. Due to Lemma 2, $\Psi_{\lambda}$ is coercive and consequently has a global minimizer. By using Theorem 1 and Proposition 5, we conclude that this minimizer is a critical point of $\Psi_{\lambda}$ and consequently a solution of problem (1). Moreover due to Lemma 3, this minimizer is nontrivial, which ends the proof. 


\subsection{An example}

Let $M(t)=\zeta+\gamma t$, where $\zeta, \gamma$ are positive constants and

$$
t:=\int_{\Omega} \frac{1}{p(x)}\left(|\Delta u|^{p(x)}+\beta(x)|u|^{p(x)}\right) d x .
$$

We first observe that

$$
M(t) \geq \zeta>0
$$

Taking $\theta=\frac{1}{2}$, we have

$$
\widehat{M}(t)=\int_{0}^{t} M(s) d s=\zeta t+\frac{1}{2} \gamma t^{2} \geq \frac{1}{2}(\zeta+\gamma t) t=(1-\theta) M(t) t .
$$

Consider the nonlocal problem

$$
\left\{\begin{aligned}
M(t)\left(\Delta_{p(x)}^{2} u+\beta(x)|u|^{p(x)-2} u\right) & =\lambda V(x)|u|^{q(x)-2} u, & & x \in \Omega \\
& u=\Delta u=0, & & x \in \partial \Omega
\end{aligned}\right.
$$

where $\beta \in L^{\infty}(\Omega)$ such that $\operatorname{essinf}_{x \in \Omega} \beta(x)>0$.

Assume that assertions of Theorem 2 or Theorem 3 are fulfilled, then any $\lambda>0$ is an eigenvalue for problem (7).

Finally, We suggest to extend the methods developed in this paper to the more general problem and in particular the one who was studied by K. Kefi and V. Rãdulescu ( See equation (2) page 440 in [21]). Moreover we suggest to extend our work on Musielak-Orlicz-Sobolev spaces (see [32, Chaper 4]) for a collection of stationary problems studied in these function spaces.

\section{Acknowledgements}

The author wish to acknowledge the approval and the support of this research study by the grant $\mathrm{N}^{o}$ CMR-2018-3-9-F-7737 from the Deanship of the Scientific Research in Northern Border University, Arar, KSA.

\section{References}

[1] S.N. Antontsev and S.I. Shmarev, A model porous medium equation with variable exponent of nonlinearity: existence, uniqueness and localization properties of solutions, Nonlinear Anal. 60 (2005), 515-545.

[2] A. Arosio and S. Panizzi, On the well-posedness of the Kirchhoff string, Trans. Amer. Math. Soc. 348 (1996), 305-330.

[3] K. B. Ali, M. Hsini, K. Kefi et al. Complex Anal. Oper. Theory. (2019). https://doi.org/10.1007/s11785-018-00885-9

[4] S. Baraket and V. Rădulescu, Combined effects of concave-convex nonlinearities in a fourth-order problem with variable exponent,Adv. Nonlinear Stud. 16 (2016), 409-419.

[5] H. Brézis, Analyse Fonctionelle, Théorie et Applications, Masson, Paris, (1983).

[6] Y. Chen, S. Levine and M. Rao, Variable exponent, linear growth functionals in image processing, SIAM J. Appl. Math. 66 (2006), 1383-1406. 
[7] F.J.S.A. Corrêa and G.M. Figueiredo, On an p-Kirchhoff equation via Krasnoselskii's genus, Appl. Math. Letters 22 (2009), 819-822.

[8] M. Dreher, The wave equation for the p-Laplacian, Hokkaido Math. J. 36 (2007), 21-52.

[9] D. Edmunds and J. Rakosnik, Sobolev embeddings with variable exponent, Studia Math. 143 (2000), 267-293.

[10] M. Eleuteri, P. Marcellini and E. Mascolo, Lipschitz continuity for energy integrals with variable exponents, Atti Accad. Naz. Lincei Rend. Lincei Mat. Appl. 27 (2016), no. 1, 61-87.

[11] X. Fan and X. Han, Existence and multiplicity of solutions for $p(x)$-Laplacian equations in $\mathbb{R}^{N}$, Nonlinear Anal 59 (2004), 173-188.

[12] A. Ferrero and G. Warnault, On solutions of second and fourth order elliptic equations with power-type nonlinearities, Nonlinear Anal. 70 (2009), 2889-2902.

[13] A. Fiscella, A fractional Kirchhoff problem involving a singular term and a critical nonlinearity. Adv. Nonlinear Anal. 8 (2019), no. 1, 645-660.

[14] Y. Fu, and Y. Shan, On the removability of isolated singular points for elliptic equations involving variable exponent, Adv. Nonlinear Anal. 5 (2016), no. 2, 121-132.

[15] M.R. Heidari Tavani, G.A. Afrouzi and S. Heidarkhani, Multiplicity results for perturbed fourth-order Kirchhoff-type problems, Opuscula Math. 37 (2017), no. 5, 755-772.

[16] M. Hsini, N. Irzi and K.Kefi, Eigenvalues of some p(x)-biharmonic problems under Neumann boundary conditions, Rocky Mountain J. Math 48 (2018), no. 8, 2543-2558.

[17] C. Ji, F. Fang and B. Zhang, A multiplicity result for asymptotically linear Kirchhoff equations, Adv. Nonlinear Anal. 8 (2019), no. 1, 267-277.

[18] K. Kefi, $p(x)$-Laplacian with indefinite weight, Proc. Amer. Math. Soc. 139 (2011), 4351-4360.

[19] K. Kefi, RACSAM (2018). https://doi.org/10.1007/s13398-018-0567-z.

[20] K. Kefi and V. Rãdulescu, On a p(x)-biharmonic problem with singular weights, Z. Angew. Math. Phys. (2017), 68:80. https://doi.org/10.1007/s00033-017-0827-3.

[21] K. Kefi and V. Rãdulescu, Small perturbations of nonlocal biharmonic problems with variable exponent and competing nonlinearities. Atti Accad. Naz. Lincei Cl. Sci. Fis. Mat. Natur. 29 (2018), 439-463. doi: 10.4171/RLM/816.

[22] K. Kefi and K. Saoudi, On the existence of a weak solution for some singular $p(x)$-biharmonic equation with Navier boundary conditions, Adv. Nonlinear Anal.8, no 1 (2019), 1171-1183

[23] G. Kirchhoff, Mechanik, Teubner, Leipzig, Germany, 1883.

[24] J.-L. Lions, On some questions in boundary value problems of mathematical physics, Proceedings of International Symposium on Continuum Mechanics and Partial Differential Equations, Rio de Janeiro 1977 (de la Penha, Medeiros, Eds.), Math. Stud., North Holland 30 (1978), 284-346.

[25] R.A. Mashiyev, S. Ogras, Z. Yucedag and M. Avci, Existence and multiplicity of weak solutions for nonuniformly elliptic equations with non-standard growth condition, Complex Variables and Elliptic Equations 57 (2012), 579-595.

[26] T.G. Meyers, Thin film with hight surface tension, Siam Rev. 40 (1998), 441-462.

[27] X. Mingqi, V. D. Rădulescu and B.Zhang, A critical fractional Choquard-Kirchhoff problem with magnetic field.Commun. Contemp. Math.21 (2019), no. 4, 1850004, 36 pp.

[28] X. Mingqi, V. D. Rădulescu and B.Zhang,Fractional Kirchhoff problems with critical Trudinger-Moser nonlinearity. Calc. Var. Partial Differential Equations 58 (2019), no. 2, Art. 57, 27 pp.

[29] X. Mingqi, V. D. Rădulescu and B.Zhang, Nonlocal Kirchhoff diffusion problems: local existence and blow-up of solutions. Nonlinearity 31 (2018), no. 7, 3228-3250. 
[30] G. Molica Bisci and D. Repovš, On doubly nonlocal fractional elliptic equations, Atti Accad. Naz. Lincei Rend. Lincei Mat. Appl. 26 (2015), no. 2, 161-176.

[31] W. Orlicz, Über konjugierte Exponentenfolgen, Studia Math. 3 (1931), 200-212.

[32] V.D. Rădulescu and D.D. Repovš, Partial Differential Equations with Variable Exponents: Variational Methods and Qualitative Analysis, Monographs and Research Notes in Mathematics, Taylor \& Francis, Chapman and Hall/CRC, 2015.

[33] M. Ružička, Electrortheological Fluids: Modeling and Mathematical Theory. Springer, Berlin (2000).

[34] M. Xiang, B. Zhang and V. D. Rădulescu, Superlinear Schrödinger-Kirchhoff type problems involving the fractional $p$-Laplacian and critical exponent. Adv. Nonlinear Anal. 9 (2020), no. 1, 690-709.

[35] A. Zang and Y. Fu, Interpolation inequalities for derivatives in variable exponent Lebesgue Sobolev spaces, Nonlinear Anal. 69 (2008), 3629-3636.

[36] V.V. Zhikov, Lavrentiev phenomenon and homogenization for some variational problems, C. R. Acad. Sci. Paris Sér. I Math. 316 (1993), no. 5, 435-439. 